\title{
THE MECHANICS OF TENSILE MEMBRANE ACTION IN COMPOSITE SLABS AT HIGH TEMPERATURES
}

\author{
A. Alskeif ${ }^{a}$, I.W. Burgess ${ }^{a}$, S.-S. Huang ${ }^{a}$ \\ ${ }^{a}$ Department of Civil \& Structural Engineering, University of Sheffield, UK
}

\begin{abstract}
The mechanics of tensile membrane action of thinlightly-reinforced concrete slabs has been reexamined during the last two years. The re-examination is based on large-deflection plastic yieldline analysis, applied to flat slabs. As deflection increases beyond the optimum yield-line pattern, tensile membrane action is mobilized and further load carrying capacity is provided. This paper represents an extension of this re-examination to include composite slabs at high temperatures. As temperature increases, the unprotected downstand steel beams significantly lose capacity, allowing for further deflection until the overall capacity degrades to the applied load. Tensile membrane action then allows further increase of steel temperature until a maximum is reached.
\end{abstract}

Keywords: Tensile membrane action, composite slab, fire, Bailey-BRE method

\section{INTRODUCTION}

Bailey \& Moore (2000a, b) presented a simplified method for the fire design of composite slabs, based on a calculation of the enhanced load capacity of the slab at large deflection due to its tensile membrane action,which was originally published by Hayes (1968). The simplified method considers that, as theunprotecteddownstand steel beams lose considerable strength at high temperature, the mechanism by which the slab resists the applied loads changes from bending resistance to tensile membrane action (TMA) at large deflection. TMA increases as the downstand steel beams lose strength, allowing for further deflection as temperature increases, until no further capacity canbe mobilized. However, the existing simplified method is based on empirical assumptions, which are made at many stages without mentioning the effects of theseassumptions. The method considers two components of load enhancement,namely bending resistance and membrane action, as independent of one another. These two independent components are eventually combined asone single enhancement, without justifying the rationality of such a process. Furthermore, the method is based on calculations which use different neutral axis depths in the $x$ and $y$ directionsfor the same cross-section, which is intuitively irrational.Burgess et al. (2013) presented an alternative simplified method based on the mechanics of TMA using the same yield-line pattern, which had been used by Bailey and Moore, but with different subsequent kinematic assumptions. This yield-line pattern has been observed in tests on loaded thin slabs which experience a full-depth central crack penetrating the slab perpendicular to its long span. The mechanism used in this paper is based on the equilibrium and kinematics presented by Burgess et al., extended to account for the presence of the unprotected downstand steel beams at high temperature, and their effects on the yield-line pattern and enhancement factor.

\section{YIELD LINE MECHANISM AND CRACKING SYSTEM EQUILIBRIUM}

As an extension of the work presented by Burgess et al. (2014), the same initial assumptions are considered in this paper. A two-way rectangular slab of aspect ratio $r$ is considered. The slab is supported vertically along its four edges, and is reinforced bytwo layers of isotropic welded mesh, which lieat its mid-surface. The slab is considered as isolated, but is in no way limited to this, or to the assumptions mentioned above. However, due to the contribution of the downstand steel beams, the resulting yield-line pattern is rotated by $90^{\circ}$ from that of a non-composite slab,due to the strength added in the original direction of the yield-line alignment. For the purpose of this paper, only one 
steel beam is considered in the study. A comparison of the usualand rotated alignments of yield-line patterns is shown in Fig. 1.

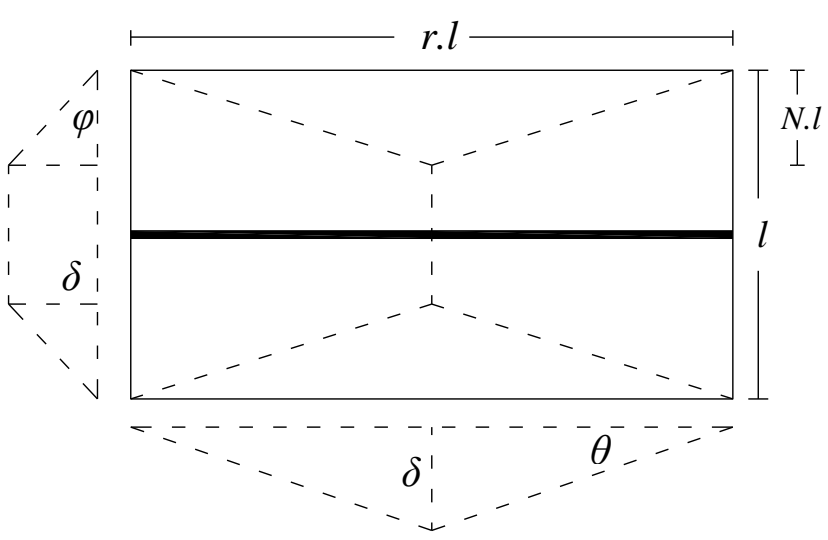

(a) Rotated Alignment

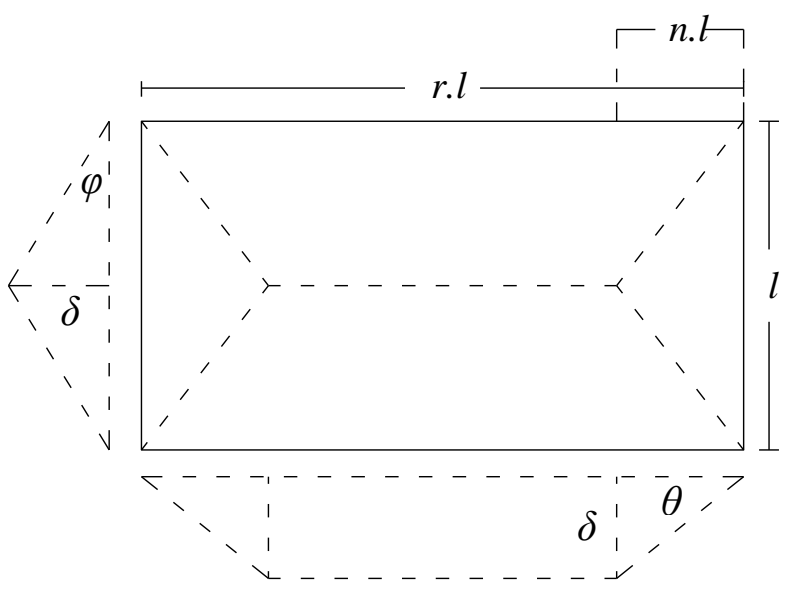

(b) Normal Alignment

Fig.1 Rotated \& normal yield-line alignments

As is the case with the normal yield-line pattern,each resultingflat facet of the slab rotates about its supporting edge by angles $\theta$ or $\varphi$, as shown in Fig. 1 . The rotation of these facets results in the same maximum deflection $\delta$ at both yield line intersection points, as shown in Fig. 2.

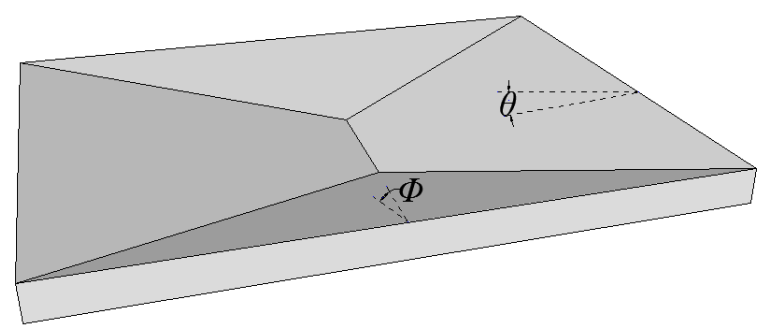

Fig.2 Rotated yield-line pattern illustrated in 3D

The optimized yield-line intersection dimensionin the presence of the steel beam, which is denoted as $N$ to distinguish the rotated from the normal alignment, is given as:

$N=-\frac{2}{3} K \cdot r^{2} \cdot l^{2}+\sqrt{\left(\frac{2}{3} K \cdot r^{2} \cdot l^{2}\right)^{2}-4 \cdot\left(\frac{2}{3} \cdot K \cdot l^{2}+\frac{4}{3} \cdot B_{c} \cdot l\right) \cdot\left(-\frac{1}{2} \cdot K \cdot r^{2} \cdot l^{2}\right)} / 2\left(\frac{2}{3} K \cdot l^{2}+\frac{4}{3} \cdot B_{c} \cdot l\right)($ Chyba!

\section{Záložka není definována.)}

Where $K$ is $2 * m_{P}\left(m_{P}\right.$ is the sagging moment capacity of the slab), and $B_{c}$ is the steel beam strength capacity. $r$ is the slab aspect ratio, and $l$ is the slab short span.This represents the level at which the yield-line capacity is minimized. It is highly unlikely that the optimum yield-line pattern will change after the yield lines have been formed. Temperature increase allows further increase of deflection due to weakening of the steel beam, allowing further capacity to be mobilized by tensile membrane action. As the deflection increases, thecompression block depth in the central region of the slab decreases until it disappears completely, whenthe whole central area is then in pure tension. During this retreat of compression from the central region of the slab, the stress blocks on the diagonal yield lines change from trapezoidal to triangular, and its depth at a slab corner can increase until the whole slab corner is in compression as shown in Fig. 3.Concrete dissipates internal work wherever slab elements are in contact. The reinforcement dissipates internal work when it is plastically stretched under tension. The extent by which the cracks between facets of the slab widen during deflection plays a crucialrole in determining the existence of both the concrete compression blocks and reinforcementtension zones. 


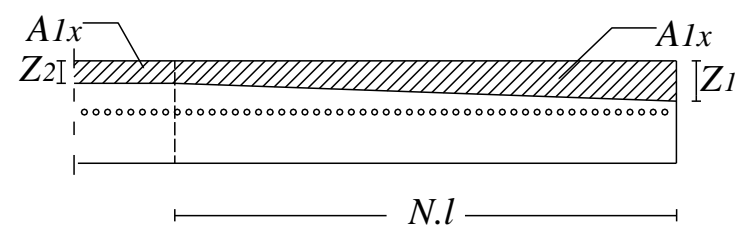

(a) Compression over the whole slab surface

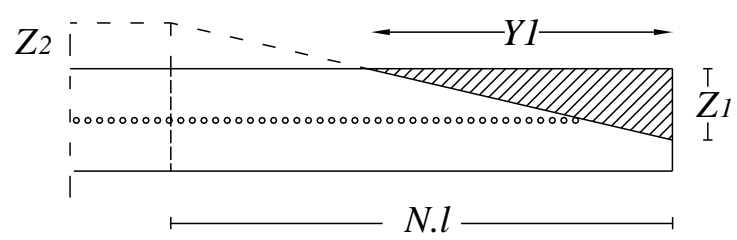

(b) Triangular compression stress block

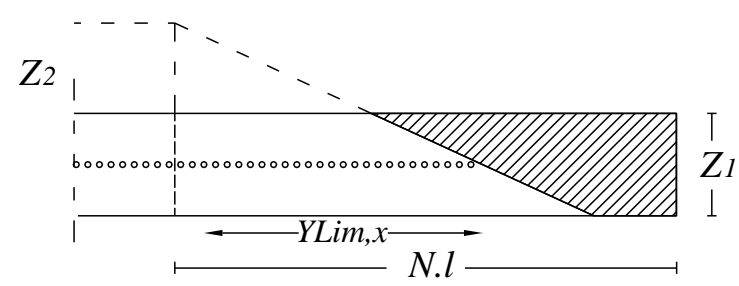

(c) Trapezoidal compression stress block

Fig.3 Compression block phases shown at the edge of the slab, as deflection increases

As shown in Fig. 3, it is possible for compression and tension to co-existover a complete yield line cross-section. However, as the crack widensthe compression zones move towards the corners and tension exists wherever the mesh lies below the neutral axis.Only reinforcement bars which are in tension are considered in the calculation of energy dissipation. Therefore, only those between any intersection with the neutral axis and the limiting distances $Y_{\operatorname{Lim}, x}$ (for X-aligned bars) and $X_{\text {Lim, }}$ (for $y$-aligned bars) at which the bars fracture are taken into account in calculation of load capacity. When the slab deflects, its facets rotate about the yield-lines by the rotation angles at its supporting edges. This results in the beams spanning the cracks to extend in a similar fashion to the reinforcement bars as shown in Fig. 4.This extension of the heated steel beams results in dissipation of plastic work which has to be included in the load capacity calculation.

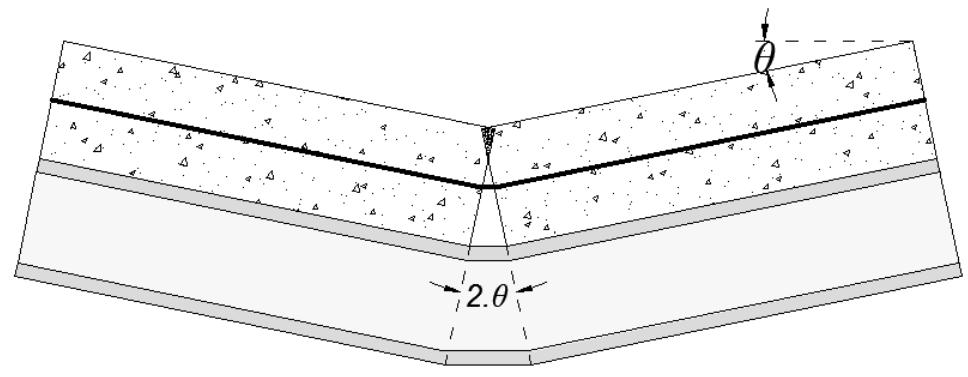

Fig.4 Extension undergone by the downstand beam, and reinforcement

Due to the compression stress blocks in the concrete, and the extensionsof the reinforcement and the steel beams, in-plane equilibrium of the slab facets must existunder the compression and tension forces crossing the yield lines. The forces involved in this equilibrium are shown in Fig. 5. $T_{x l}$ and $T_{x 2}$ represent reinforcement tension forces on Elements 1 and 2 respectively in the x-direction. $T_{y l}$ is the reinforcement tension force in the y-direction. $T_{b}$ is the tension force generated by the extension of the downstand beam. $C \& C_{x 2}$ are the resultant concrete compression forces and $S$ represents the resultant shear force between facets on a diagonal yield line. When the central yield line opens as the slabs facets lose contact, $C_{x 2}$ disappears abruptly, leaving only the pure reinforcementtensile force across this yield line. Along the diagonal yield-lines, this complete separation does not occur, 
but it develops progressively, starting from the intersection point of the yield-lines, towards the corner of the slab, effectively "unzipping" the mesh.

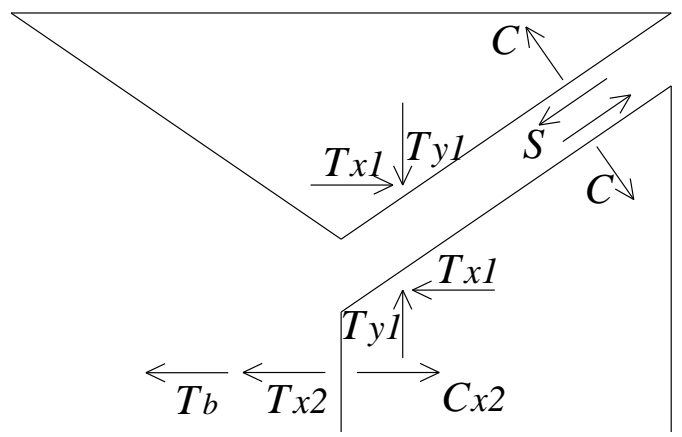

Fig.5 Force equilibrium on the slab elements

Eliminating the shear force $S$, the equilibrium state can be expressed by the equation:

$$
T_{y 1} \cdot \operatorname{Cos}(\gamma)+\left(T_{x 1}+T_{x 2}+T_{b}\right) \cdot \operatorname{Sin}(\gamma)=C_{x 2} \cdot \operatorname{Sin}(\gamma)+C(1)
$$

In which the forces are given by:

$$
\begin{aligned}
& T_{y 1}=\left(X_{L i m, y}-X_{T}\right) \cdot f_{p y} \\
& T_{x 1}=\left(Y_{L i m, x}-Y_{T}\right) \cdot f_{p x} \\
& T_{x 2}=l \cdot(1-2 \cdot N) \cdot f_{p x} \\
& C=A_{1 x} \cdot f_{c} / \operatorname{Sin}(\gamma) \\
& C_{x 2}=A_{2 x} \cdot f_{c}
\end{aligned}
$$

The terms $\left(X_{\text {Lim, } y^{-}} X_{T}\right)$, and $\left(Y_{\text {Lim }, x^{-}} Y_{T}\right)$ are the distances over which the reinforcement bars are still intactand generating their full yield strength. $f_{p x}$ and $f_{p y}$ are reinforcement forces in both directions. $A_{1 x}$ and $A_{2 x}$ are the compression block areas, as illustrated in Fig. 3, earlier.

\section{KINEMATICS OF THE SOLUTION}

The forces, which were calculated above, are substituted into the equilibrium equation and expressed as a function of the horizontal movement in they-direction $\Delta_{y} . \mathrm{Y}$ is taken as the major direction in the calculations since it is the direction of the obtained rotated yield-line pattern. This horizontal movement $\Delta_{y}$ changes as deflection increases.A program has been coded inMatlab to conduct a deflection-controllediteration. At each deflection level, the temperature is increased, until the slab capacity has degraded to the level of the applied load. This allows further deflection until a maximum load capacity is reached and the slab is considered to have structurally failed.

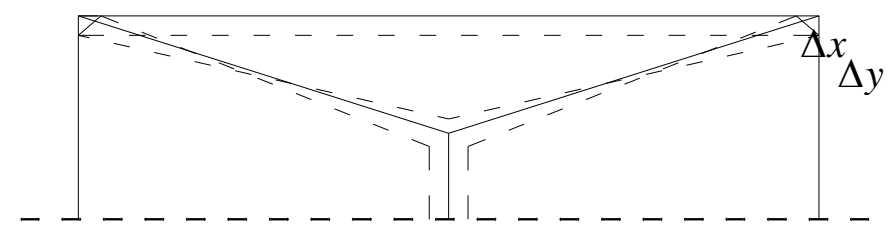

Fig.6 Horizontal movements seen from the top surface of the slab

The calculated forces and geometry allow the calculation of the internal energy dissipated within the slab, which allows thecalculation of the load capacity of the slab $P$. The internal plastic work of the reinforcement is expressed using the plastic stretch of the bars across the widening crack width between the slab elements. As for concrete areas which are still in contact, the internal energy is expressed in terms of the compression forces between the overlapped elements. The loss of potential energy of the transverse loading on the surface of the slab $P$ is calculated similarly to the optimal small-deflection yield-line mechanism, and is given as:

$$
W_{\text {ext }}=P \cdot r \cdot l^{2} \delta \cdot\left(\frac{1}{2}-\frac{1}{3} N\right)
$$


Where: $W_{\text {ext }}$ is the external work done by loads; $\delta$ is the maximum deflection of slab.According to plastic energy theory, external work done $=$ internal energy dissipated. This gives the load capacity of the slab as:

$$
P=W_{\text {int }} /\left[r \cdot l^{2} \delta \cdot\left(\frac{1}{2}-\frac{1}{3} N\right)\right]
$$

Where: $W_{\text {int }}$ is the internal work of the slab.The iteration increases the temperature of the unprotected steel beams until this calculated load capacity $P$ has reduced to the applied load. Then the deflection loop is repeated. This iterationcontinues until no further increase is possible. The enhancement is taken in terms of the temperature calculated at the end of every internal loop.

\section{APPLICATION OF THE METHOD}

A composite slab will be considered in order to demonstrate the application of the newly extended method. The slab used in the Matlab model is $9 \mathrm{~m}$ by $6 \mathrm{~m}$ with an aspect ratio $r=1.5$; thickness $t=130 \mathrm{~mm}$; reinforced by isotropic A142 mesh $\left(142 \mathrm{~mm}^{2} / \mathrm{m}\right.$ in both directions) located at the slab mid-depth; The mesh bar spacing is $200 \mathrm{~mm}$. Reinforcement ductility $=5 \%$; Yield stress of reinforcement $f_{y s}=500 \mathrm{MPa}$; Beam section (UKB 406x140x39); Yield stress of the steel beam $f_{y}=355 \mathrm{MPa}$; Concrete characteristic strength $f_{c}=35 \mathrm{MPa}$. Uniformly distributed transverse loading on the slab $=4 \mathrm{kN} / \mathrm{m}^{2}$. The result of the analysis is shown in Fig. 7 below.

(a) Temperature Enhancement

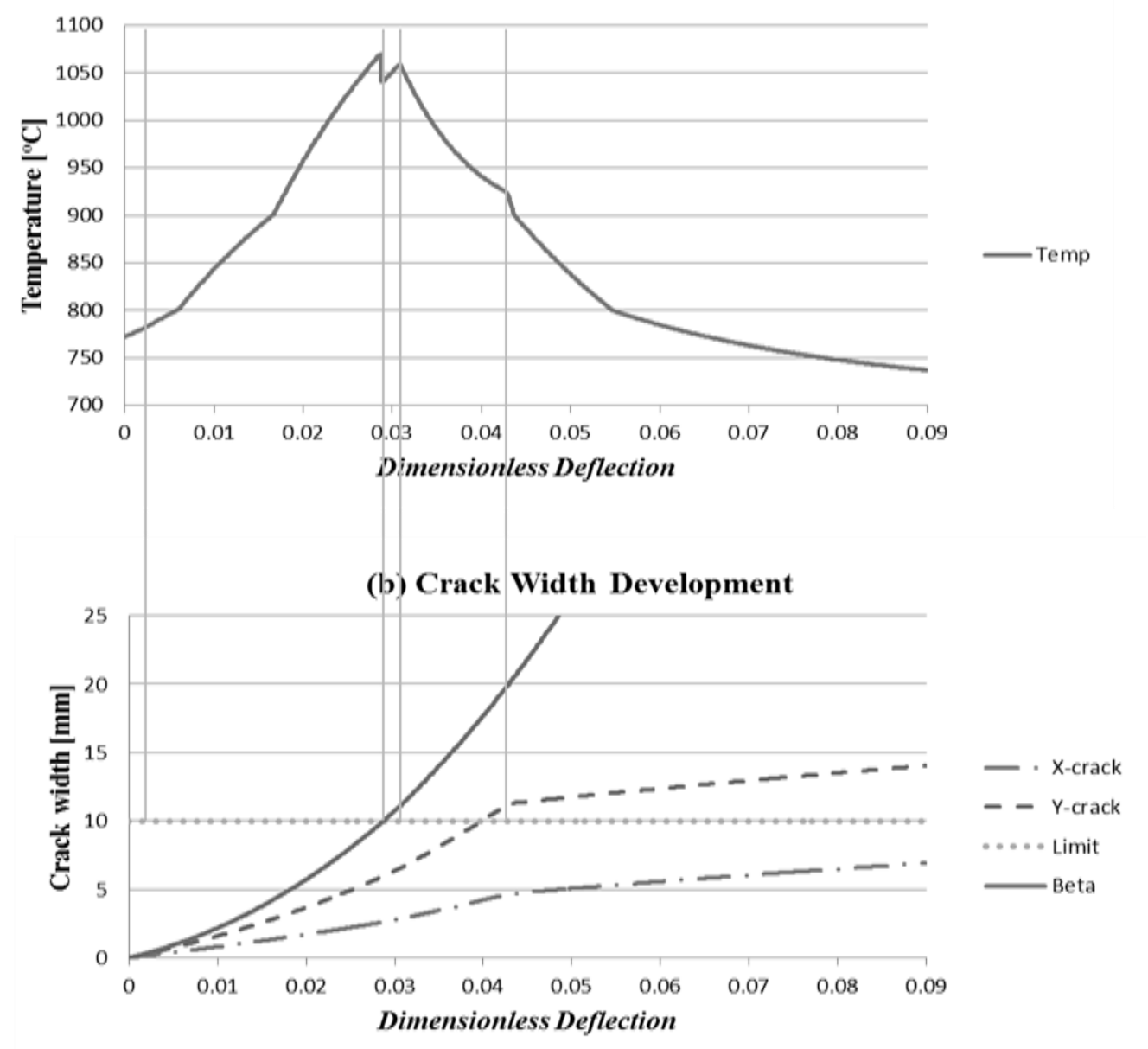

Fig.7: (a) represents temperature against deflection; (b) represents crack width development against deflection. The vertical lines define phase changes through the process

Fig. 7(a) shows the enhancement in terms of temperature against normalized deflection. Fig.7(b) shows the development of the crack widths of the diagonal yield-lines in the $x$ and $y$ directions. It also monitors the development of the central yield-line crack width $\beta$. The limit crack width, 
demonstrated by a horizontal line,represents the limit at which reinforcement fractures. The first vertical line in Fig. 7(b) represents the end of the first phase at which the compression stress block becomes triangularon the diagonal yield lines of the slab, while compression disappearsfrom the central yield line. The end of second phase witnesses the fracture of the mid yield-line bars,coinciding with a temperature decrease which gives the first peak shown in Fig. 7(a). The final two phases account for the unzipping of the bars in the $y$-direction and then $x$ and $y$, respectively.However, if it happens that the slab cools down before any of thetemperature enhancement peaks, shown in Fig. 7(a), has been reached, the slab will survive with its current reinforcement and crack status, and the rest of the chart will simply be theoretical in this case. If, on the other hand, the temperature happens to rise higher than the temperature enhancement peak for the same slab configurations, the slab fails due to excessive temperature and the rest of the enhancement chart does not apply anymore.Similarly, the slab forces, shown in Fig. 8 below, which have been illustratedin thinner lines after the second peak, account for the theoretical course of action which might change in case the factual fire scenario differs from the theoretical one.

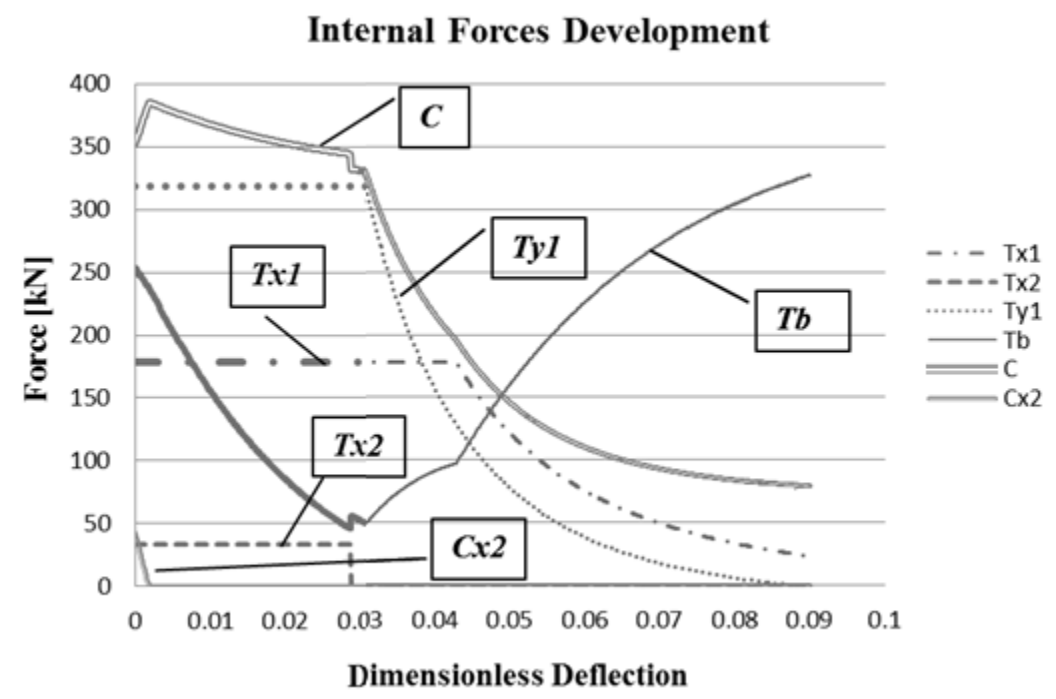

Fig.8 Internal forces on the slab facets

\section{CONCLUSIONS}

This paper has demonstrated an extension of the alternative simplified method which accounts for composite slabs with unprotected downstand steel beams at high temperature. The analysis conducted in this study is based on plastic yield-line mechanism at large deflection. The temperature is assumed only to affect the unprotected steel beams since concrete has low thermal conductivity, which causes the unprotected steel beams to heat faster, leaving the reinforcement at a relatively cool temperature. Temperature increase controls the enhancement, accounting for the reinforcement fracture that occurs when the slab experiences large deflections, as the unprotected steel beams lose capacity. The enhancement depends on the temperature to which the slab is subjected, anddifferent fire scenarios will affect the actual enhancement.

\section{REFERENCES}

Bailey, C.G. \& Moore, D.B., 2000. The structural behaviour of steel frames with composite floors slabs subject to fire-part 1 Theory. Struct Eng, 78, pp.19-27.

Burgess, I.., Huang, S. \& Staikova, S., 2014. A re-examination of the mechanics of tensile membrane action in composite floor slabs in fire. In 8th International Conference on Structures in Fire. Shanghai, China.

Burgess, I.W., Dai, X. \& Huang, S., 2013. An alternative simplified model of tensile membrane action of slabs in fire. ASFE 2013, Prague.

Hayes, B., 1968. Allowing for Membrane Action in the Plastic Analysis of Rectangular Reinforced Concrete Slabs. Magazine of Concrete Research, 20(65), pp.205-212. 